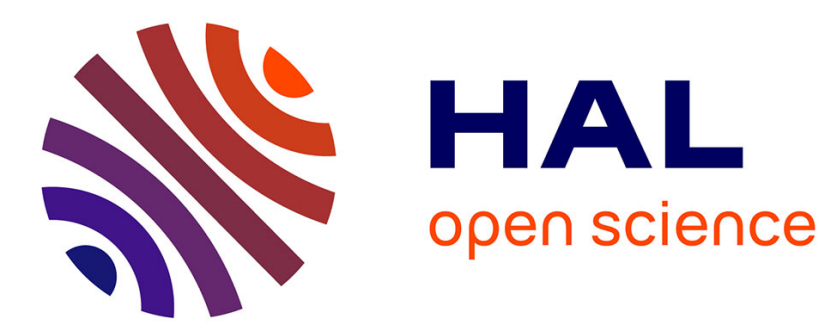

\title{
The implication of the lattice in the non-equilibrium behaviour of a glassy crystal
}

Michel Descamps, Jean-François Willart, G. Odou, K. Eichhorn

\section{To cite this version:}

Michel Descamps, Jean-François Willart, G. Odou, K. Eichhorn. The implication of the lattice in the non-equilibrium behaviour of a glassy crystal. Journal de Physique I, 1992, 2 (6), pp.813-827. 10.1051/jp1:1992181 . jpa-00246603

\section{HAL Id: jpa-00246603 https://hal.science/jpa-00246603}

Submitted on 1 Jan 1992

HAL is a multi-disciplinary open access archive for the deposit and dissemination of scientific research documents, whether they are published or not. The documents may come from teaching and research institutions in France or abroad, or from public or private research centers.
L'archive ouverte pluridisciplinaire HAL, est destinée au dépôt et à la diffusion de documents scientifiques de niveau recherche, publiés ou non, émanant des établissements d'enseignement et de recherche français ou étrangers, des laboratoires publics ou privés. 
Classification

Physics Abstracts

$61.50-64.70 \mathrm{P}-61.40 \mathrm{D}$

\title{
The implication of the lattice in the non-equilibrium behaviour of a glassy crystal
}

\author{
M. Descamps ( $\left.{ }^{1}\right)$, J. F. Willart (1), G. Odou ( $\left.{ }^{1}\right)$ and K. Eichhorn $\left({ }^{2}\right)$ \\ (1) Laboratoire de Dynamique et Structure des Matériaux Moléculaires $\left(^{*}\right)$, Université de \\ Lille 1, Bât P5, F 59655 Villeneuve d'Ascq, France \\ (2) DESY - F 41, Hasylab, Notkestr. 85, D 2000 Hamburg 52, Germany \\ (Received 5 February 1992, accepted in final form 17 March 1992)
}

\begin{abstract}
Time resolved X-ray measurements on single crystals of the mixed system (cyanoadamantane) ${ }_{1-x}$ (chloroadamantane) ${ }_{x}$, for $x \cong 0.25$, have been made. Special attention has been paid to the underlying lattice in the glassy state. The rotational glass transition is signalled by a jump of the expansivity at $T_{\mathrm{g}}$ as in conventional glasses. There is no signature of the freezing on the peak profiles after a deep quench below $T_{\mathrm{g}}$. In the neighbourhood of $T_{\mathrm{g}}$, there is evidence of a slow correlated amplification of antiferroelectric and ferroelastic fluctuations respectively observed at the $\mathrm{X}$ and $\Gamma$ points of the Brillouin zone. In this quasi static ordering process the average underlying lattice is preserved but the mean square displacements are considerably increased.
\end{abstract}

\section{Introduction.}

The study of glasses and of the glass transition is currently very active for two main reasons :

- In the domain of conventional glasses this is mainly due to the recent development of a mode coupling theory [1]. The latter has reactivated the discussion between a purely dynamic interpretation of the glass transition and the research of a masked thermodynamic singularity occurring at a temperature somewhere below that of the calorimetric glass transition $\left(T_{\mathrm{g}}\right)$ [2-4]. In these systems, the translational degrees of freedom are mainly involved. In experiments this can be seen as a source of difficulty in so far as the structural information only appear through a structure factor $S(|\mathrm{Q}|)$ which is spatially averaged.

- During the past few years the domain of study has been extended to a larger class of disordered systems. These are in particular crystalline phases with an orientational disorder of certain entities which are expected to be the only ones involved in the freezing process. For this reason they have been seen as suitable models of conventional glasses. In fact this family includes very miscellaneous situations which may be related only indirectly to the conventional glass state.

The most formerly studied of these glassy crystalline systems are the magnetic spin glasses [5]. A prerequisite to their formation is a «positional » disorder obtained by dilution which

$\left.{ }^{*}\right)$ UA CNRS 801. 
leads to a distribution of the exchange interactions. Contrary to conventional glasses, frustration - and freezing as well - is induced by this dilution.

The second category is that of « orientational glasses" a review of which was presented recently [6]. These are mixed molecular crystals showing a paraelectric or paraelastic phase at high temperatures. The necessity of dilution is the aspect they share with the magnetic glasses. Here too, this dilution can induce a frustration effect [7]. However, their proper character seems to be mainly associated with the coupling between the orientational degrees of freedom and the lattice displacements. This mediation of the lattice in the freezing mechanism observed at low temperatures appears in every class of orientational glasses. It is the case with mixed crystals of $\mathrm{NaCN}: \mathrm{KCN}$ [8] where the dilution does not involve the rotors (CN): the cations of different sizes induce static random strains. It is also the case in systems like $\mathrm{KBr}$ : $\mathrm{KCN}$ [9] where the dilution directly acts at the rotor level.

Some connections with the dynamical description of conventional glasses have been established by Michel [10] in a theory which does not consider the frustation phenomena as essential. Through a particular mode coupling formalism, he was able to describe freezing as a collective mechanism leading to a non-ergodic instability. The foundation of the theory is however the coupling of orientations with the random strain fields generated by the substitutional disorder. The glass transition can happen if this coupling is not too strong with regard to the coupling of orientations to lattice vibrations. As a result, the orientational and translational modes are frozen together. In diffraction experiments the signature of this coupling below the glass transition is an apparent broadening of the Bragg peaks (B.P.) of the underlying lattice [9].

In this article, we are interested in the third category which is that of « glassy crystals » [11]. They are obtained by the undercooling of the orientationally disordered phase of molecular crystals (platic phases). Contrary to the systems previously described, the dilution of an impurity is not at all necessary to get the orientational freezing. On the other hand there is a similarity with the glass-forming liquids, both in the way the glassy state is reached and in the manifestation of the glass transition. This concerns in particular the following points which are not found in the diluted orientational glasses:

- The disordered phase must be undercooled below the first order equilibrium phase transition (occurring at $T_{\mathfrak{t}}$ ) leading to the ordered phase. This transition is not removed - as it is by the dilution in the orientational glasses - but bypassed : the success of the quench depends on a kinetic condition, which is to cross rapidly the temperature range of rapid transformation (the nose in a TTT diagram [12]). The glass transition occurs in a range of temperatures far below $T_{\mathrm{l}}$.

- The transition from an ergodic to a non-ergodic situation appears in a jump of specific heat at $T_{\mathrm{g}}$ with change of temperature.

The study of this kind of glasses is very attractive since we can expect them to be the rotational analogue of conventional glasses, and thus contributes to the understanding of the latter. The underlying lattice provides a good opportunity of following with accuracy the evolution of the spatial correlations through $S(Q)(Q-V e c t o r$ rather than $|Q|)$ in diffraction experiments. Of course on can use this opportunity only if single crystals of the plastic phase can be quenched. To our knowledge, this could be achieved only in two cases : cyclohexanol [13] and cyanoadamantane ( $\mathrm{CN}-\mathrm{a})[14,15]$.

From a technical point of view the difficulty is that one must quench a sample which is effectively plastic and often sublimates quickly. It is thus necessary to place the crystal in a glass capillary. Under strong cooling the contact of the crystal to the glass wall may induce cracks in the single crystal. 
Here we present some results of a study of $\mathrm{CN}-\mathrm{a}$ and mixed compounds of the latter with the chloroadamantane $(\mathrm{CN}-\mathrm{a})_{1-x}(\mathrm{Cl}-\mathrm{a})_{x}$, for which the above difficulties does not occur. At room temperature these compounds have isomorphous f.c.c. orientationally disordered structures [16]. For $x \leqslant 0.5$, they show the same tendency to form a glassy crystal after quenching [19]. However the mixed compounds with $x \cong 0.25$ are found to be better glass formers in the sense which is usually given for liquids. As a consequence the metastable state ranging between $\left(T_{\mathrm{t}} \cong 237 \mathrm{~K}\right)$ and $\left(T_{\mathrm{g}} \cong 165 \mathrm{~K}\right)$ is more easily investigated in particular in diffraction experiments. In figure 4 , for instance, two measurements of lattice parameters could be performed in the domain of metastability for temperatures higher than $T_{\mathrm{g}}(180 \mathrm{~K}$ and $190 \mathrm{~K}$ respectively). At these temperatures the metastable state of the mixed compound has a life time long enough to allow investigations of more than $30 \mathrm{~min}$.

Aging effects have been clearly seen in an X-ray investigation of $\mathrm{CN}-\mathrm{a}$ [15]. They appear in the slow growth of superstructure peaks at the Brillouin zone boundary ( $X$ point) which reflect an antiferroelectric ordering of the molecules. In the course of the specific investigation of the ordering kinetics, some reference measurements performed at the zone center ( $\Gamma$ point) pointed out that aging also induced an apparent broadening of the Bragg peaks (B.P.). This result prompted us to reinvestigate these B.P. more specifically both in time and temperature after quenchs from the high temperature disordered phase. We are in particular concerned with a possible implication of the lattice in the glass transition mechanism. From the results obtained with the orientational glasses it is a fundamental point which could guide any further theoretical approach.

The technique used is conventional $\mathrm{X}$-ray diffraction supplemented by some information drawn from the high spatial resolution of a synchrotron source.

The paper is organized as follows.

The experimental procedures are described in section 2 . In section 3 , we consider the possible influence of the glass transition on the lattice properties. Next (Sect. 4) aging effects are investigated.

\section{Experimental details.}

The single crystals of $(\mathrm{CN}-\mathrm{a})_{l_{-x}}(\mathrm{Cl}-\mathrm{a})_{\lambda}$ with $x \cong 0.25$ have been grown by slow evaporation of a solution in methanol (crystal size $\cong 0.8 \times 0.8 \times 0.8 \mathrm{~mm}^{3}$ ). Experiments were performed with a conventional $X$-ray source equipped with a pyrolitic graphite monochromator ( $\lambda$ Mo $K \bar{\alpha}=0.711 \AA$ ). The samples were mounted on the Eulerian circle of an automatic diffractometer allowing rapid changes of orientations to follow the simultaneous time evolution of several reflections and superstructure peaks. The sample temperature $T$ could be controlled to within $0.5 \mathrm{~K}$ in the range $110 \mathrm{~K}<T<300 \mathrm{~K}$. Quenching was achieved by blowing a stream of cool nitrogen over the sample. We estimate the time for quenching from room temperature (R.T.) to be less than $5 \mathrm{~s}$ using this method. In the experiment the crystal was not protected by a capillary. The only precaution is not to stay too long at R.T. in order to avoid sublimation. Some test experiments were performed on the synchrotron source DESY with single crystals of the same origin (Diffractometer D3 of the Hasylab).

The results presented in this paper were obtained on the same single crystal at several stages of a treatment which implies the temperature and the time. An overall picture of the thermal history imposed to the sample is shown in figure 1. The partition of the experiment is as follows :

a) study of the domain of stability of the disordered phase : measurements of the lattice parameters and peak profiles;

b) study of the peak profiles after a deep quench below the glass transition $T_{\mathrm{g}}$; 
c) measurements of the lattice parameters below $T_{g}$;

d) aging experiment at $160 \mathrm{~K}$ : time evolution of the B.P. and superstructure peaks;

e) measurements of the lattice parameters above $T_{\mathrm{g}}$ in the domain of metastability of the disordered phase. The sample was recycled to R.T. after every quench in order to avoid parasitic nucleation.

The $\theta-2 \theta$ scans presented in the paper (Figs. 3, 7, 9) are not corrected for $\mathrm{K}_{\alpha 2}$ spectral line. This leads, at large $Q$, to non-significant asymmetry and shifting with respect to the $\Delta \theta=0$ setting valid for the $\mathrm{K}_{\alpha 1}$ radiation. $\mathrm{K}_{\alpha 2}$ contribution is included in the fitting procedures.

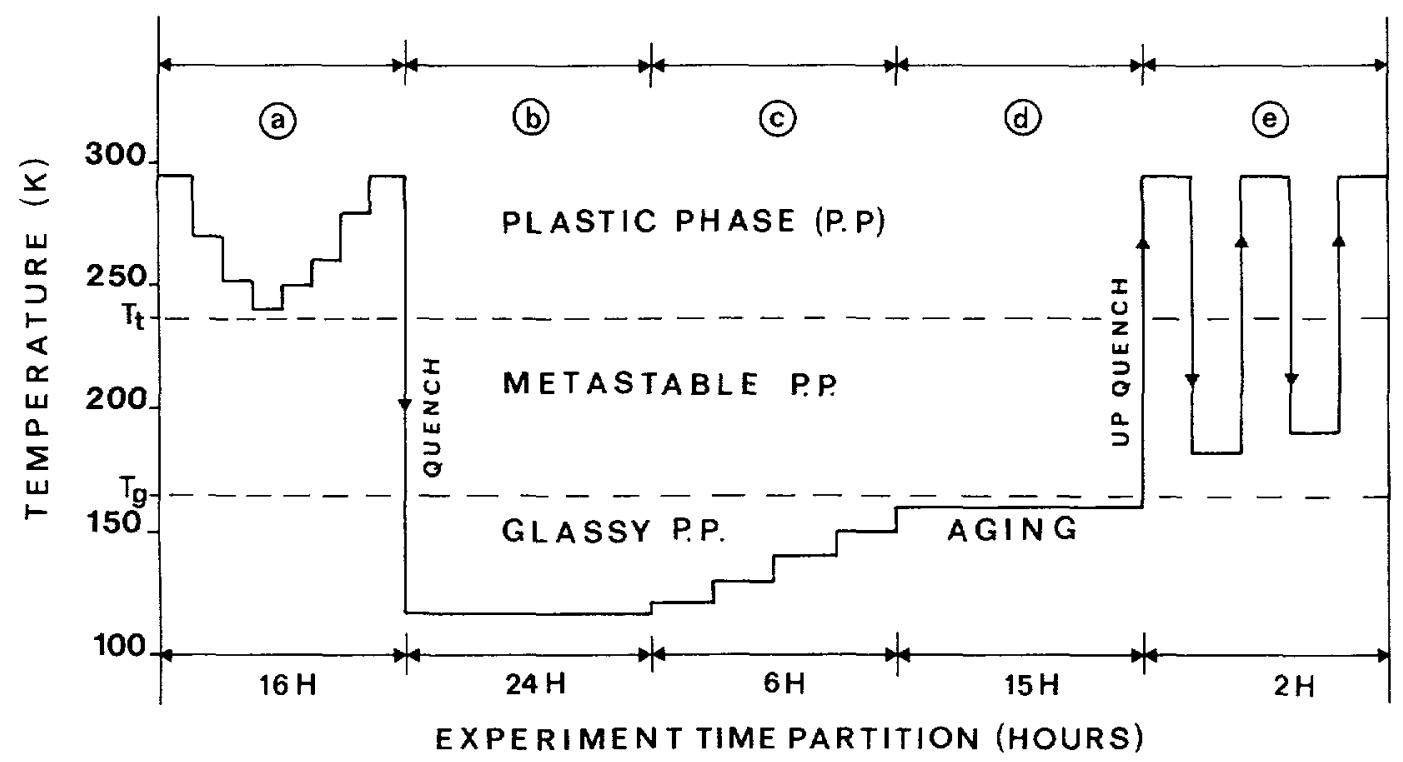

Fig. 1. - Thermal history of the experiment. The five stages appearing on the diagram are described in the text.

\section{The cubic lattice in equilibrium and undercooled conditions.}

A remarkable feature is the perfection of the crystals of the disordered phase. This was tested with the synchrotron source and is illustrated in figure 2 . On several crystals, $\omega$-scans as well as $\theta-2 \theta$ scans of the (511) B.P. gave widths ranging between $0.004^{\circ}$ and $0.007^{\circ} \mathrm{HWHM}$ in $\theta$. It is typically the width observed on this instrument for a perfect silicon crystal. The measurements were useful in order to determine the instrumental resolution of the conventional apparatus, used in the following ; the same scan gave a width of $0.06^{\circ} \mathrm{HWHM}$ in $\theta$.

The same crystal could be studied in the domain of equilibrium within the plastic phase $(T \geqslant 240 \mathrm{~K})$ and under undercooled conditions (Fig. 1). It was not quenched to temperatures ranging between $190 \mathrm{~K}$ and $\cong 237 \mathrm{~K}$ where the transformation to the low temperature phase is the most rapid. At R.T. it was found to be cubic f.c.c. The lattice constants were obtained from the set of equivalent (511) B.P. giving ( $\left.a=9.833 \AA \pm 0.002 ; \alpha=90^{\circ} \pm 0.02^{\circ}\right)$. Figure 3a presents $\theta-2 \theta$ scans through various reciprocal lattice points. The Bragg intensity decreases considerably at higher hkl but, contrary to other, more disordered, plastic crystals, several orders of reflection can be clearly observed. When corrected for the $\mathrm{K}_{\alpha 2}$ contami- 


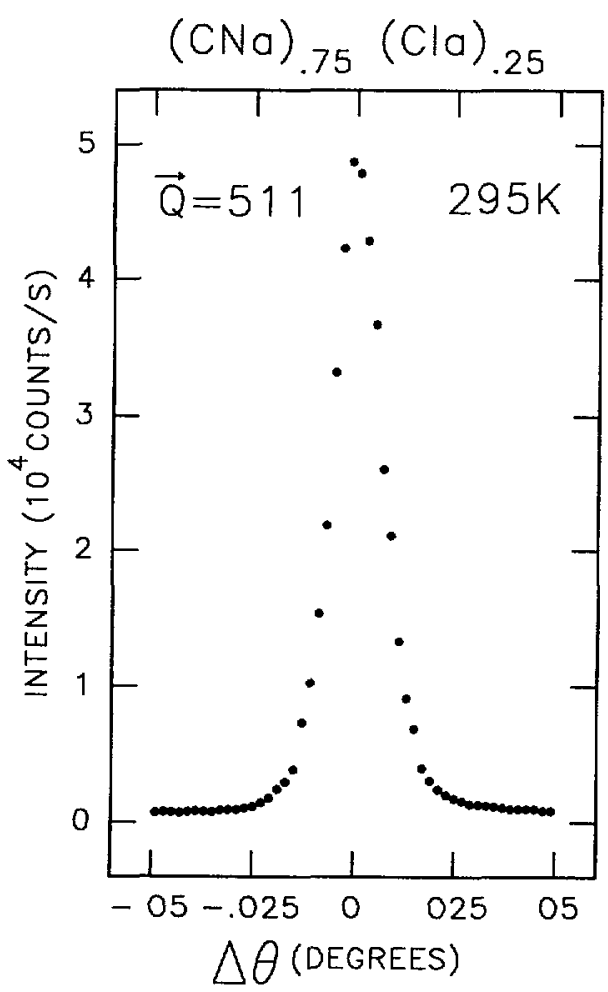

Fig. 2. $-\omega$ scan of the (511) Bragg peak recorded at R.T. with a synchrotron source $\left(H W H M \cong 0.007^{\circ}\right.$ in $\theta$ ).

nation and plotted on the $Q$ scale, it becomes apparent that there is no $Q$ dependent broadening. As a consequence, the large orientational disorder of the molecules acts as a distortion of the first kind [17a] and is functionally indistinguishable from the effect of thermal vibrations.

It will be shown in section 5 that aging the crystal at temperatures neighbouring $T_{\mathrm{g}}$ involves an apparent broadening of the B.P. Our aim at present is to know if such a broadening can be detected soon after a quench. To suppress aging effects, the crystal was quenched at a temperature well below $T_{\mathrm{g}}$ where the kinetics of evolution are imperceptible. Figure $3 \mathrm{~b}$ shows scans which were performed after a quench at $116 \mathrm{~K}$. Neither splitting nor broadening of the lines can be detected. The widths of the peaks remain those of the instrumental resolution. The lattice is found to be again f.c.c. with the same accuracy on the parameters (at $116 \mathrm{~K} a=9.652 \AA \pm 0.002 \AA ; \alpha=90^{\circ} \pm 0.02^{\circ}$ ). We only notice a sizeable increase of the peak intensity.

The evolution with temperature of the cubic lattice parameter was obtained from the (511) B.P. during the stages $a, c$, e of the experiment described in figure 1 . The result is given in figure 4. The experimental points are clearly divided into two data sets which can be independently fitted to two straight lines. Note that the point corresponding to $160 \mathrm{~K}$ departs slightly from the fitted line. This can be explained by some unavoidable aging effects occurring quite rapidly at this temperature (cf. Sect. 4). The parameters measured in the metastable domain $(180 \mathrm{~K}, 190 \mathrm{~K})$ of the plastic phase follow the line of the high temperature data.

The intersection of the lines at $\cong 166 \mathrm{~K}$ gives rise to a pronounced jump of the expansivity $\Delta \alpha \cong 10^{-4} \mathrm{~K}^{-1}$ (Fig. 5). Such an expansivity change is a thermodynamic signature of the glass 


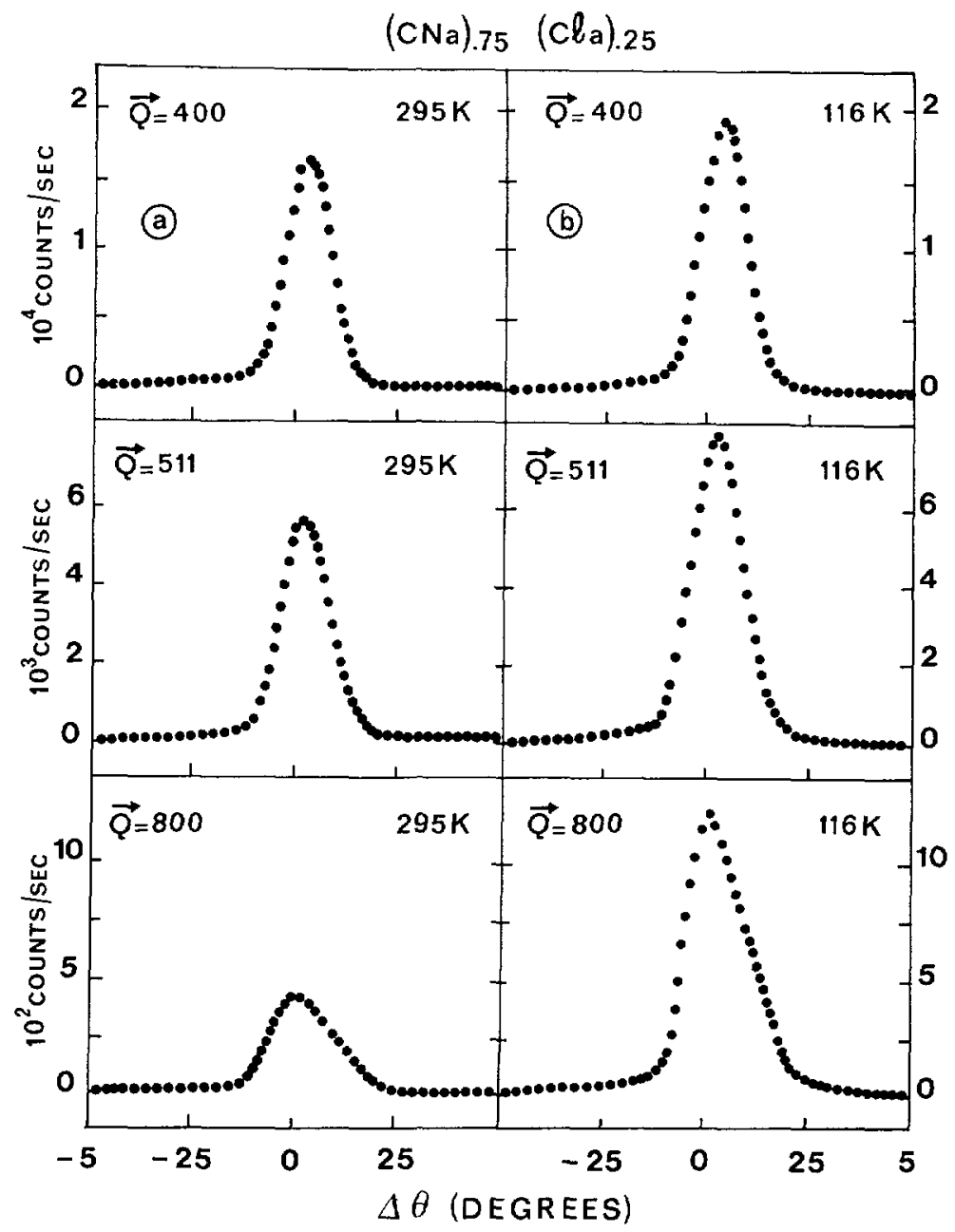

Fig. 3. $-\theta-2 \theta$ scans of different Bragg peaks at R.T. a) and just after a quench b) into the glassy plastic phase at $116 \mathrm{~K}$. In the limit of the instrumental resolution, no broadening of the lines was detected in this quench experiment ( $K_{\alpha 2}$ contaminants are not removed).

transition $T_{\mathrm{g}}$ [18]. Its occurrence at a temperature somewhat below that of the pure $\mathrm{CN}-\mathrm{a}$ [19] reflects the slightly shorter time scale for the molecular dynamics in the mixed system.

One can conclude that, while the manifestation of the conventional glass transition occurs at $T_{\mathrm{g}}$, no noticeable change is observed below $T_{\mathrm{g}}$ at the Brillouin zone center, which could indicate a modification of the lattice strains. This is true after a deep quench and at the short times after a more shallow quench.

\section{Aging effects at the Brillouin zone center.}

At low temperatures, roughly for $T<140 \mathrm{~K}$, no clear evolution of the $\mathrm{X}$-ray spectra are visible at least during the first hours following a quench. Towards higher temperatures (140 $\mathrm{K}<T<T_{\mathrm{g}}$ ) large time dependent effects have been signalled for the $(x=0)$ pure compound $[14,15]$. In a previous study a slow growth of diffuse scattering around the 


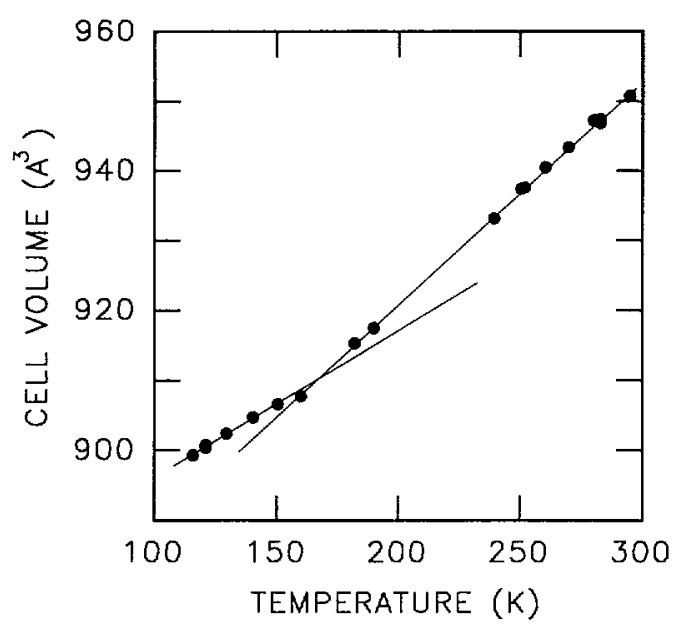

Fig. 4.

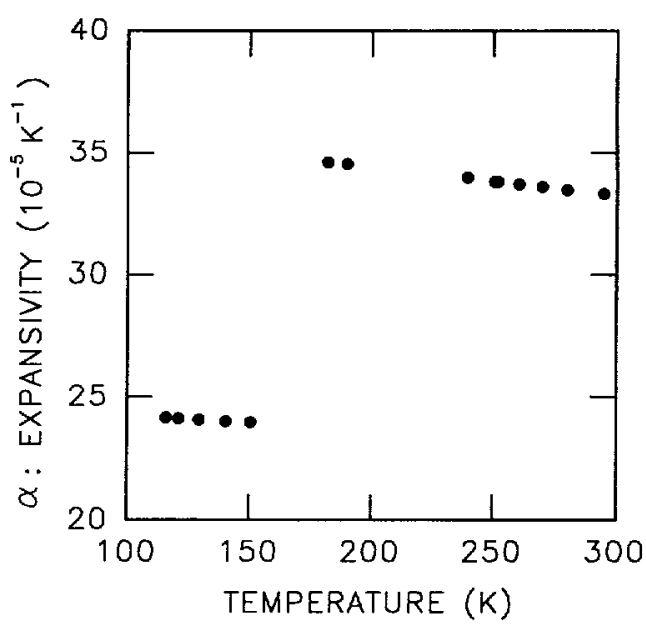

Fig. 5.

Fig. 4. - Temperature dependence of the cell volume. The data corresponding to the stable and metastable plastic phase and those corresponding to the glassy plastic phase are independently fitted to two straight lines.

Fig. 5. - Coefficient of thermal expansion : $\alpha=\frac{1}{V}\left(\frac{\partial V}{\partial T}\right)$ versus temperature.

forbidden reflections of the f.c.c. structure was observed. Figure 7 shows that these Brillouin zone boundary effects (X points) also appear during an isothermal aging of the $(x=0.25)$ compound. At the same temperature the only change between $x=0$ and $x=0.25$ is the slightly higher rate of evolution in the latter case ; the general outlook of the growth curve (Fig. 8c) is preserved i.e. immediate - but slow - growth and gradual slowing down which gives a weak time dependence to the overall process.

Furthermore some test measurements of the main B.P. of $\mathrm{CN}$-a have shown changes occurring at the Brillouin zone center ( $\Gamma$ points). A summary of the results will be given before describing our specific investigation of this phenomenon on the mixed compound. These results are represented in figures $6 a, 6 b$, taken from a previous paper [15].

- The lattice parameter determination repeatedly performed on the (511) B.P. indicates an apparent contraction of the cubic cell resulting from a slow shifting of the top of the peaks toward larger $\mathbf{Q}$ values.

- Scans through various B.P. taken at certain moments during an aging run at $160 \mathrm{~K}$ display a broadening of the lines which can be significant after several tens of hours especially at large values of $Q$.

- The broadening $\Delta Q$ seems to be very dependent of the $Q$ value $\left(\Delta Q \propto Q^{n}\right.$ with $n \geq 2$ ). This could point out the occurrence of crystalline imperfections of the second kind [17a]. These would induce large fluctuations of the intermolecular distances which destroy the average crystalline periodicity.

However the points quoted above do not match easily with other observations :

i) the loss of translational invariance has been found to be perfectly reversible upon reheating ;

ii) while being only signalled by a limited number of experimental points, the broadening seems to obey kinetics of evolution which have characters in complete opposition to those 


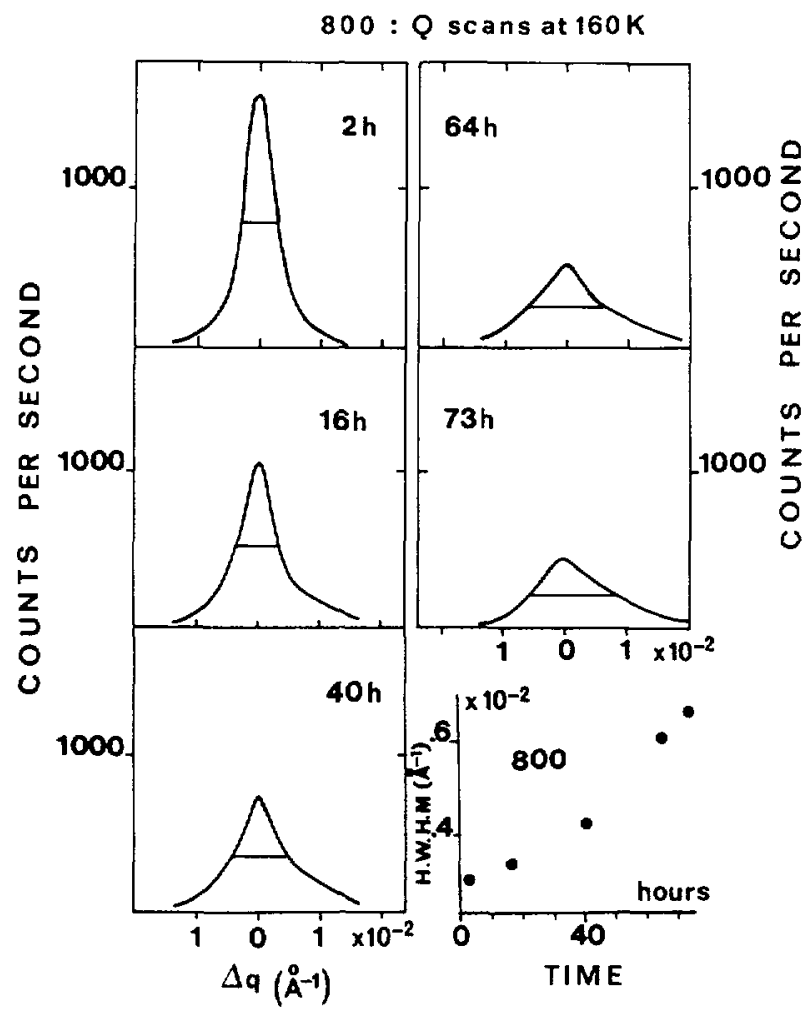

a)

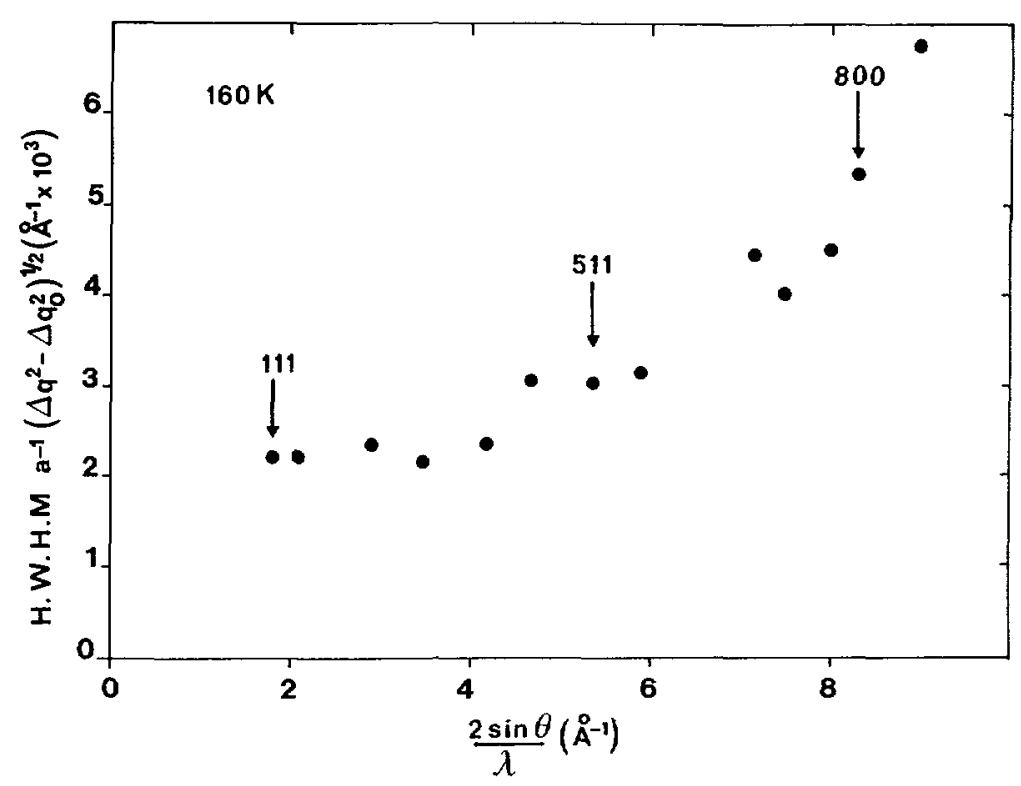

b)

Fig. 6. - a) $Q$ scans through the (800) reflection at various aging times at $160 \mathrm{~K}$. HWHM is in $10^{-2} \AA^{-1}$. (CN-a) from reference [15]. b) Peak broadening as a function of momentum transfer $Q$ after aging for 64 hours at $160 \mathrm{~K}$. ( $\mathrm{CN}-\mathrm{a})$ from reference [15]. 


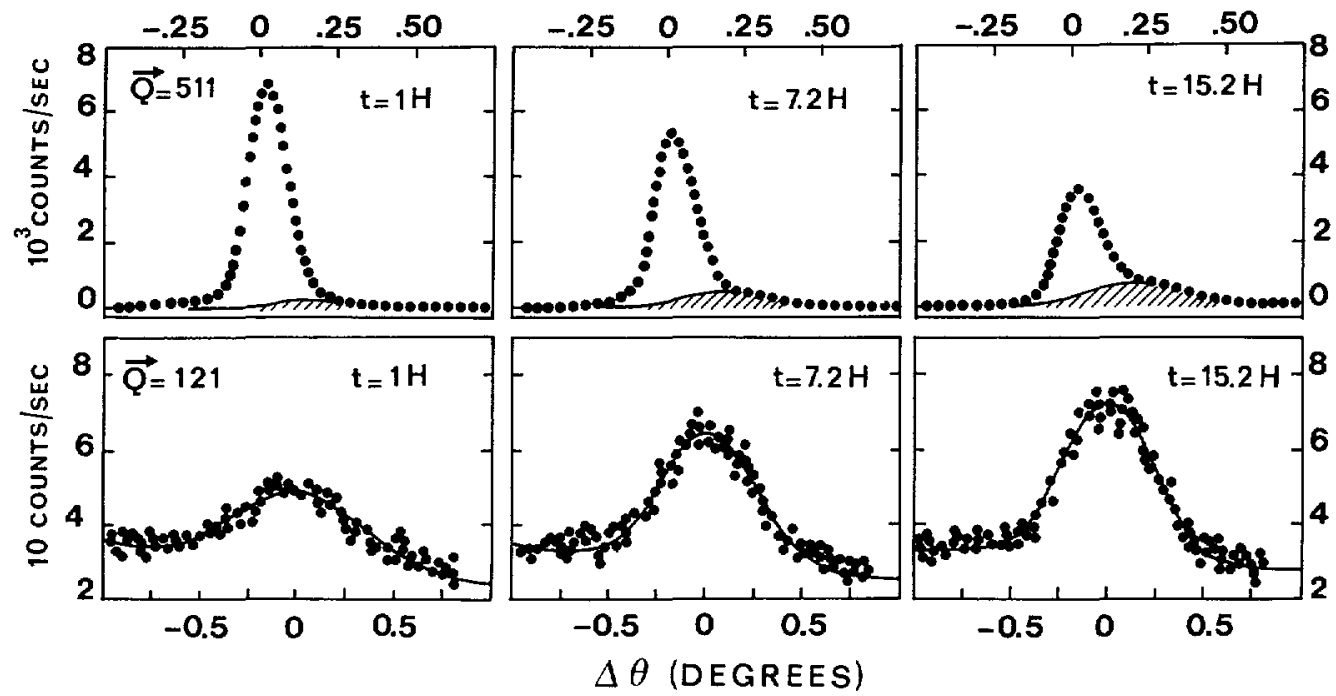

Fig. 7. - Time evolution of the (511) Bragg peak and the (121) superstructure peak after a quench to $160 \mathrm{~K}$ ( $\theta-2 \theta$ scans). The hatched area corresponds to a diffuse contribution growing in the tails of the Bragg peak ( $K_{\alpha 2}$ contaminants are not removed).

observed at the zone boundary : one notes an acceleration of the broadening when the growth of the superstructure peaks seemingly approaches saturation;

iii) a careful examination of the $a(t)$ curve shows the same tendency with an initial plateau of about 10 hours.

Important information is revealed by the present analysis of the evolution of the lines performed on the compound ( $x=0.25$ ) from the start of an aging run at $160 \mathrm{~K}$. The vicinity of the (511) node and the superstructure peak (121) were scanned in an alternating way during 15 hours. The results are summarized in figure 7 . A close inspection of the shape of the B.P. in this first stage of the evolution clearly reveals a double structure which was not suspected in the previous experiments. At each time, the overall profile can be quantitatively described by the sum of a Gaussian peak which has the width of the initial B.P. and a broader, smaller, diffusive component (D.C.) which is found in the wings towards the higher Bragg angles. It cannot be fitted to a Lorentzian but can be reasonably well described by a Gaussian while decreasing slightly more rapidly in the tails.

The time dependence of this double peaked diffraction profile was followed through 25 scans. The following features became apparent :

First, the D.C. increases and is combined with a decrease of the intensity of the B.P. Figures 8a-b illustrate this time dependence. In 15 hours the ratio of the two components changes by a factor of about 6 . This obviously leads to an apparent broadening of the overall line with time.

It is difficult to extract very precise information concerning the evolution of the widths of the two components. However it is apparent that all along the aging process, the width of the B.P. remains about that of the resolution (HWHM $\cong 0.06^{\circ}$ in $\theta$ ). The width of the D.C. is significantly larger but almost constant or very weakly time dependent only ( $\mathrm{HWHM} \cong 0.134^{\circ}$ in $\theta$ ). A slow, limited increase of the $\theta$-offset between the two components ( $\delta \theta=0.026^{\circ}$ after 15 hours) is noticed which contributes to make the profile more asymmetric. 


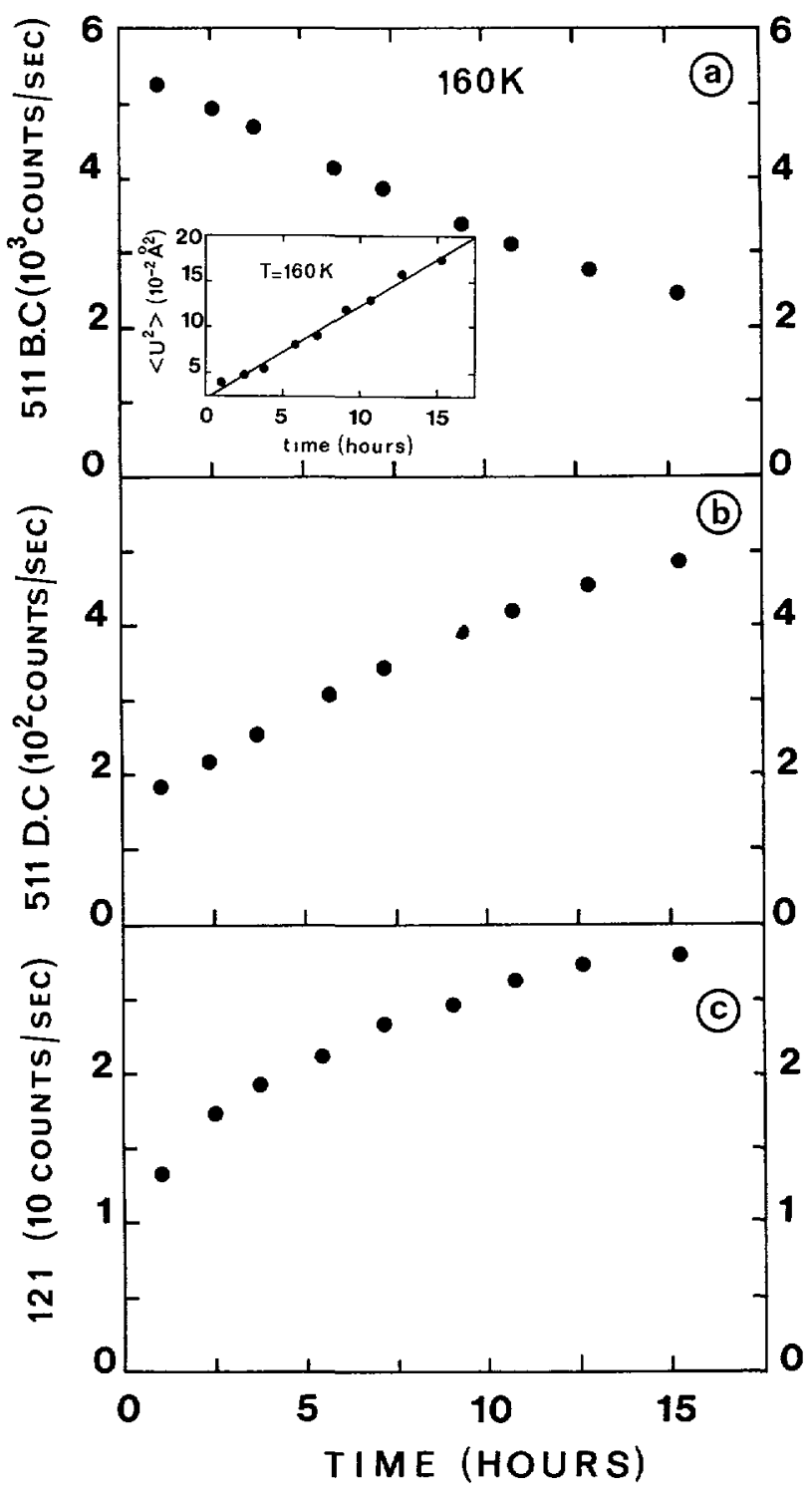

Fig. 8. - Kinetics of evolution observed at the $\Gamma$ (511) and $\mathrm{X}$ (121) points during a 15 hours aging at $160 \mathrm{~K}$. a) peak intensity of the 511 Bragg component (inset: time evolution of the mean square displacement), b) peak intensity of the 511 diffuse component, c) peak intensity of the 121 superstructure peak.

The $Q$ dependence is illustrated in figure 9, which shows scans at the (400) and (800) positions performed at the start and at the end of the aging run. Comparing the different scans, it is clear that the ratio of the diffuse over the Bragg component as well as its rate of evolution increase with increasing $Q$. This effect is so important at the $(800)$ position that the Bragg reflection becomes nearly obscured: after 15 hours the intensity of the D.C. is $\cong 0.7$ times that of the B.P. Fitted values of the peak parameters are listed in table I.

The large decrease of the Bragg intensity is coherently explained by the decrease of an effective Debye Waller factor. As shown in figure $8 \mathrm{a}$ the associated mean-square displacement 
Table I. - Fitted value of peak intensity corrected from the $K_{\alpha 2}$ radiation.

\begin{tabular}{|c|c|c|c|c|}
\hline Peaks & $I_{\mathrm{DC}}(t \cong 0)$ & $I_{\mathrm{BC}}(t \cong 0)$ & $I_{\mathrm{DC}}(t \cong 15 \mathrm{~h})$ & $I_{\mathrm{BC}}(t \cong 15 \mathrm{~h})$ \\
\hline $121(\mathrm{X})$ & 13 & & 28 & \\
\hline $400(\Gamma)$ & 205 & 13891 & 852 & 8333 \\
\hline $511(\Gamma)$ & 178 & 5220 & 481 & 2416 \\
\hline $800(\Gamma)$ & 43 & 802 & 165 & 115 \\
\hline
\end{tabular}

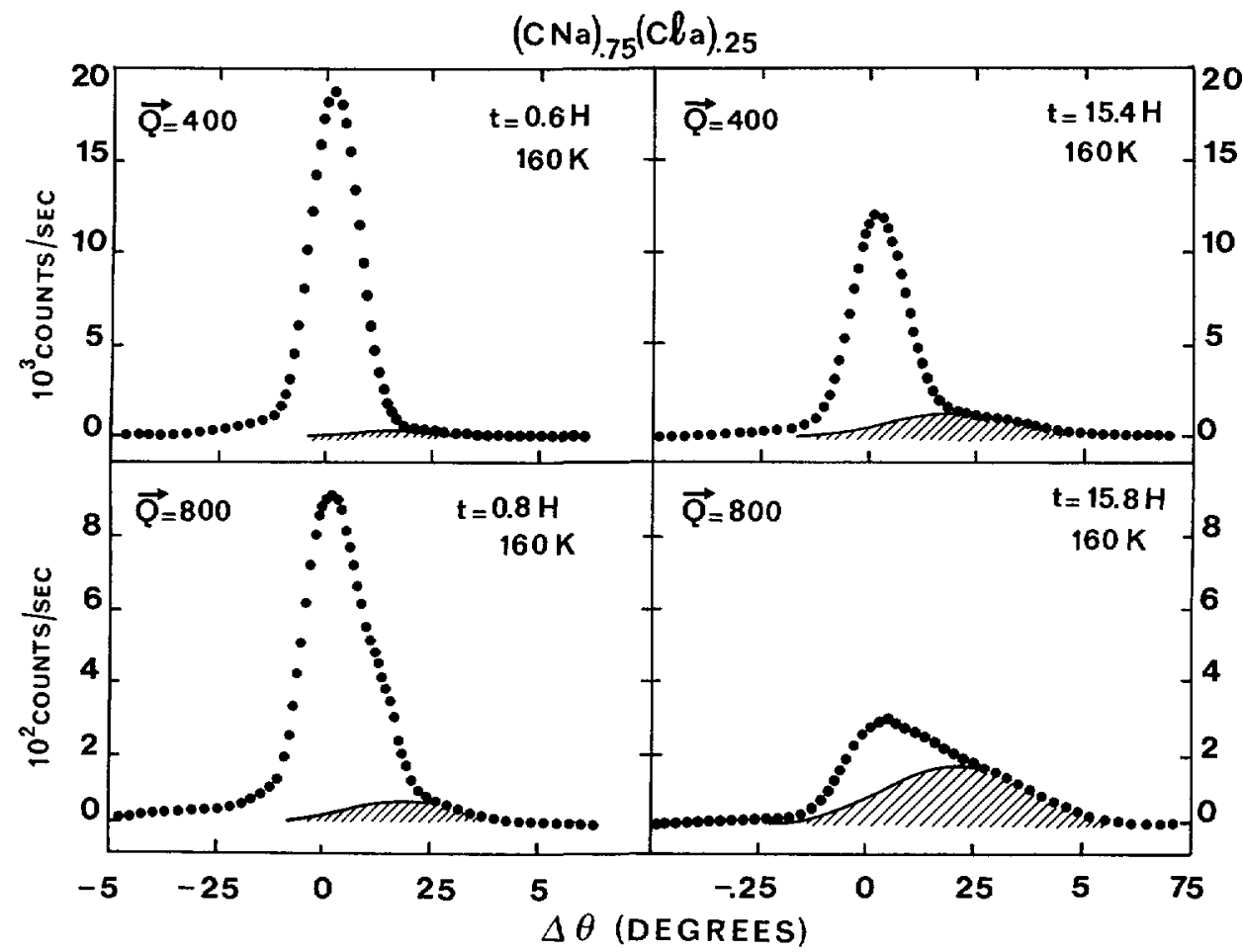

Fig. 9. - Profiles of the (400) and (800) Bragg peaks ( $\theta-2 \theta$ scans) at the beginning and at the end of a 15 hours aging at $160 \mathrm{~K}$. The hatched areas corresponds to a diffuse contribution growing in the tails of the Bragg peaks ( $K_{\alpha 2}$ contaminants are not removed).

increases almost linearly in time. At the end of aging it is 2.5 times that observed at R.T. On the other hand the widths of the D.C. and their $\theta$-offset from the corresponding B.P. are seemingly independent of $Q$. Their rate of increase upon aging no longer shows a clear $Q$ dependence.

The important point revealed by figures 7,8 is that the intensity of the D.C. grows together with the diffracted intensity observed at the $X$ point. The corresponding growth curves can be nearly superimposed which shows the identity of the kinetics. 
Discussion. - Satellites occurring at the $X$ points indicate a tendency to form a superstructure. The diffusive peaks have been found to be static by neutron scattering measurements on $\mathrm{CN}$-a [20]; thus they represent an ordering of the molecular dipoles. Considering the behaviour of the ordering kinetics under such a deep undercooling and in particular the fact that the response is immediate and not delayed, it was concluded in [15] that the mechanism is most probably a continuous ordering rather than a droplet nucleation. In real space this may be described as the spontaneous development of diffuse regions of increasing antiferroelectric order throughout the disordered lattice. The situation is analogous to that of spinodal decomposition for a demixing system but, in this case, it involves and order parameter which is not conserved [21]. Accordingly the mechanism is the rotational diffusion of the molecules rather than the volume diffusion which operates in the conserved case.

The non-randomly distributed perturbations of the parent cubic lattice are also the centers of elastic distortions which are probed at the $\Gamma$ point of the Brillouin zone. The growth at this point of the D.C. in exact correlation with that observed at the $\mathrm{X}$ point can be interpreted as being the amplification of Fourier components of strain waves [17b]. The width of the D.C. gives an estimate of the minimum value of the lattice modulation wavelength which can grow. It appears to be about 2 times that of the minimum wavelength for the orientational modulations.

The asymmetry of the D.C. with regard to the average lattice node points out a correlation between the orientational and positional disorder [17c]. According to the direction of this asymmetry one thus expects that the most diffusive lattice planes are also the less spaced.

Molecules of adamantane derivatives are achieved by introduction of a bulky side group into the adamantane molecule and are much less globular than adamantane itself. It has been shown [22] that steric repulsions between the Van der Waals envelopes of the molecules play an important part in the formation of the crystalline field and also in the formation of strong orientational correlations. One can suspect that the rotational translational coupling is generated by steric hindrance as shown schematically in figure 10 .

From X-ray experiments one cannot determine whether the ordering is static or dynamic, but due to the correlations mentioned above, the D.C. seems to be quasistatic. From the evolution of the B.P. it appears that the crystal remains cubic on an average. The time dependence of the Debye-Waller factors could be explained by the amplification of the quasistatic translational modes. This leads to large anomalous mean-square displacements : a situation which is close to that described by the theory of Michel and Rowe [26]. An experiment performed on a much longer aging time would be very suitable in order to correlate more precisely the time dependence of the different data.

\section{Summary and conclusion.}

The most relevant results of this $\mathrm{X}$-ray investigation of the Brillouin zone center of $(\mathrm{CN}-\mathrm{a})_{1-{ }_{x}}(\mathrm{Cl}-\mathrm{a})_{\lambda}$ are the following ones.

- Effect on the lattice of the change-over to the glassy-crystalline state. Contrary to what was observed in $\mathrm{KBr}$ : $\mathrm{KCN}$ orientational glasses [8] no effective broadening of the fundamental lines of the underlying cubic lattice can be observed, at least within the limit of resolution of the conventional apparatus which was used. This negative result is important since it could give a strong indication that the coupling of orientations with both translations and random strains, as introduced in the mode coupling theory of the diluted orientational glasses [10], either would not be fundamental to explain the entrance of the present system into a non-ergodic situation, or would have more subtle manifestations. Anyway, the nature of the «defects» which could induce static strains is not clear in the present system. In 

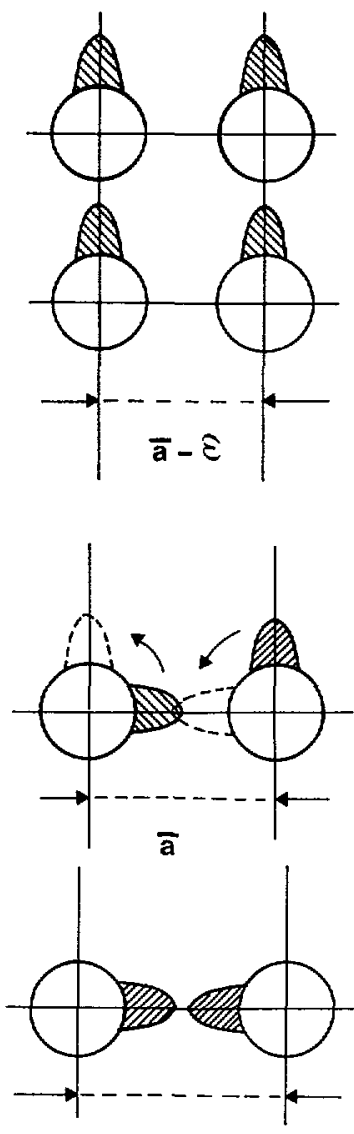

$\overline{\mathbf{a}}+\varepsilon$

Fig. 10. - Schematic illustration of a possible mechanism for translation-rotation coupling in $\mathrm{CN}$-a.

particular one should not cite the dilution of $\mathrm{Cl}-\mathrm{a}$ since the pure $\mathrm{CN}-\mathrm{a}$ has an exactly similar glass behaviour. On the other hand the expansivity of the lattice shows a pronounced change at $T_{\mathrm{g}}$. This raises the question of the connection between the process of falling out of equilibrium of the assembly of rotors and the apparent decrease of anharmonicity.

- Effect on the lattice of an aging process below $T_{\mathrm{g}}$.

Under isothermal aging at not too low a temperature, an apparent broadening of the B.P. slowly takes place. It is shown in this paper that, in fact, it corresponds to the correlated growth of a diffuse component slightly shifted from the $\Gamma$ position and to the drop of the B.P. intensity itself. Both effects are correlated to a slow development of superstructure peaks situated at the zone boundary ( $X$ point).

This is interpreted as a process of ordering which occurs in a continuous rather than nucleated way. The mechanism is the slow, but spontaneous amplification of strongly coupled orientational antiferroelectric and ferroelastic fluctuations. In this process the crystal remains cubic on average but shows an effective increase of the mean-square displacements associated with the strain field. The amplification of homophase fluctuations essentially proceeds in coherency with the parent lattice. This is in perfect agreement with the complete reversibility observed when heating up again. 
In [15] it has been shown that the kinetics of antiferroelectric ordering is extremely weakly time dependent. For example, the characteristic size $L$ provided by the radial width of the $\mathrm{X}$ superlattice peaks grows as $L=A t^{b}$ where $b \cong 0.1$. Here $t$ is the time elapsed after the quench. In a system with a non-conserved order parameter one expects $b=1 / 2$ [23]. The steric hindrance between rotating molecules has been proposed as a mechanism which could explain this unusual value of $b$. The simulation of a simple [24] model including steric constraints could effectively give, during a transient, values of $b$ somewhat lower than the expected one but not small enough to explain the experimental results. Because of the coupling which has been shown above it seems reasonable to assume that the anomalous slowing down of the kinetics could also have its origin in the need to accommodate elastic strains as the transformation proceeds. Theoretical predictions on the time dependence would then have to introduce explicitly the translation rotation coupling $[25,26]$.

Since the ordering kinetics are not delayed after a deep quench, an extremely limited amount of homophase fluctuations must certainly spread out during the quench itself. It cannot be discarded that these fluctuations influence the freezing. They could play the role of pre-existing «defects » although they are certainly not randomly distributed in the lattice. This would lead to very fine effects : the quenched phase is worth being studied at higher resolution; the quality of the crystals would allow such an experiment.

\section{Acknowledgements.}

This work was supported by the EEC Science contract SC1-0426-C (CD). We thank Dr J. Naudts and Prof. K. H. Michel for useful discussions.

\section{References}

[1] Gotze W., Aspects of structural glass transition, in Liquids Freezing and the Glass transition, J. P. Hansen, D. Levesque and J. Zinn Justin, Eds. (North Holland, 1990).

[2] Angell C. A., J. Phys. Chem. Solids 49 (1988) 863.

[3] Souletie J., J. Phys. France 51 (1990) 883.

[4] Sethna J., Shove J. D., Huang M., Phys. Rev. B 44 (1991) 4943.

[5] Binder K., Young A. P., Rev. Mod. Phys. 58 (1986) 801.

[6] Hochli U. T., KNorr K., LoIDl A., Adv. Phys. 39 (1990) 405-615.

[7] Carmesin H. O., Binder K., Z Phys. B 68 (1987) 375.

[8] LoIdl A., SChräder T., BOHMER R., KNORR K., KJems J. K., Born R., Phys. Rev. B 34 (1986) 1238.

[9] Loidl A., Knorr K., Rowe J. M., Mc Intyre G. F., Phys. Rev. B 37 (1988) 389.

[10] Michel K. H., Z. Phys. B, Cond. Mat. 68 (1987) 259 ; Bostoen C., Michel K. H., Phys. Rev. B 43 (1991) 4415.

[11] SugA H., SeKi S., J. non Cryst. Sol. 16 (1974) 171.

[12] El Adib M., Descamps M., Chanh N. B., Phase transitions 14 (1989) 85.

[13] Ceccaldi D., Denoyer F., Lambert M., Szwarc H., J. Phys. France 41 (1980) L365 ; André D., Ceccaldi D., Szwarc H., J. Phys. France 45 (1984) 731.

[14] Descamps M., Caucheteux C., Odou G., Sauvajol J. L., J. Phys. Lett. 45 (1984) L719.

[15] Descamps M., Caucheteux C., J. Phys. C 20 (1987) 5070.

[16] Foulon M., Lefebvre J., Amoureux J. P., Muller M., Magnier D., J. Phys. France 46 (1985) 919 ;

Foulon M., Thèse de Doctorat d'Etat, Lille (1987).

[17] GuINiER A., Théorie et technique de la radiocristallographie (Dunod, Paris, 1964) a) pp. 490-492 ; b) pp. $539-541$; c) pp. $588-591$.

[18] Angell. C. A. and Sichina W., Ann. NY Ac Sc 279 (1976) 53-67. 
[19] Foulon M., Amoureux J. P., Sauvajol J. L., Lefebvre J., Descamps M., J. Phys. C : Solid State Phys. 16 (1983) L265.

[20] Lefebvre J., Rolland J. P., Sauvajol J. L., Hennion B., J. Phys. C : Solid State Phys. 18 (1985) 241.

[21] Binder K., Rep. Prog. Phys. 50 (1987) 783.

[22] Descamps M., J. Phys. C : Solid State Phys. 15 (1982) 7265.

[23] Allen S. M., Cahn J. W., Acta Met. 27 (1979) 1085.

[24] Willart J. F., Naudts J., Descamps M., Phase Transition 31 (1991) 261.

[25] Michel K. H., NaudTS J., Phys. Rev. Lett. 39 (1977) 212.

[26] Michel K. H., Rowe J. M., Phys. Rev. B 32 (1985) 5827. 